\title{
Field-Distribution's CAD and Characteristics of Electrostatic Immersion Objective
}

\author{
Xingquan Wang \\ School of Physics and Electronic Information; Institute \\ of Optoelectronic Materials and Technology \\ Gannan Normal University \\ Ganzhou 341000, China \\ E-mail: wangxingquan813@163.com
}

\section{Fengpeng Wang}

School of Physics and Electronic Information; Institute of Optoelectronic Materials and Technology

Gannan Normal University

Ganzhou 341000, China

E-mail: wangfengpeng@163.com

\author{
Jun Huang \\ School of Physics and Electronic Information; Institute \\ of Optoelectronic Materials and Technology \\ Gannan Normal University \\ Ganzhou 341000, China \\ E-mail: hj.9518@163.com
}

Shoucai Yuan

School of Physics and Electronic Information; Institute of Optoelectronic Materials and Technology

Gannan Normal University

Ganzhou 341000, China

E-mail: ysc810369@sina.com

\begin{abstract}
Based on the numerical calculation method, a program is designed to solve the field distributions and equipotential lines in a electrostatic immersion objective. Then the characteristics are discussed. Successive approximation method is used to solve the finite difference equation of field distributions and the scan-line searching method is used to solve the coordinate of equipotential lines. Results from the program show that the rotationally symmetric electric field is characterized by nonuniformity so that the electron beam can be focused and imaged. Also axial potential distributions are nonlinear to produce a radial force and equipotential lines are distributed in a saddle on the nearby axial extreme point of potential. The program offers a exact and effective way to design the electrostatic immersion objective.
\end{abstract}

Keywords-electrostatic immersion objective; successive approximation method; field-distribution; potential; CAD

\section{INTRODUCTION}

A charged particle beam is concerned in many major research fields of science and technology $[1,2]$, such as the electronic beam devices widely used in scientific research and the microwave vacuum devices used in the exploration of the universe, satellite communications, navigation and tracking. A variety of electron spectrometers [3, 4], mass analyzers [3, 4] and electron microscopys [5] which are necessary to do the basic science research are related to the forming and focusing on charged particle beams. Also it exists in modern electronic and ion beam micromachining technology, in high energy particle accelerator, in controlled thermonuclear reaction. In the promotion of these continuous development of production practice and scientific experiments, it is required to explore how to control the movement of charged particles by using electric and magnetic fields.

Electrostatic immersion objective is widely used in electron guns, image tubes, image intensifiers and other electronic optical systems[6-8]. A controllable fine electron beam with the directional projection and focusing forming is the major working substance in a variety of electrical vacuum devices as well as electron microscopes, electron beam exposure machines, and so on.

To analyze and design various electronic optical, the methods often are simulating devices, model test or numerical calculation[8-10]. For the electrostatic immersion objective, only similar results can be got and it is difficult to value the imaging quality and set the related parameters by the various kinds of existing simulating devices due to the limits of accuracy or other reasons. By the model test of the removable electronic optical, the effects of various factors together are generally believed to be closer to the reality. However, by this method it is not only difficult to analyze the effect of an individual fact and determine an individual aberration, but also the achieving results still differ from the actual situations. In addition, due to the longer period for an experiment, the method is often taken with other way cooperatively.

By numerical calculation method high accuracy can be achieved, and various aberrations and even the electron optical transfer function can comprehensively be calculated. However, it fails to be applied due to restrictions on the level of technology in the past. Now due to the development of the fast electronic computer numerical calculation has being the accurate and effective method for analyzing and designing the imaging system of the electrostatic immersion objective.

\section{NUMERICAL CALCULATIONS OF FIELD DISTRIBUTIONS AND EQUIPOTENTIAL LINES}

\section{A. Finite difference equation}

Potentials of electrostatic field satisfy the Laplace equation with $\nabla^{2} \Phi=0$ in the absence of space charge. In the electron optics, the electrostatic field is often a two dimensional one including the flat field and the axial rotational symmetric field. However, there are different 
forms of difference because of the different form of Laplace equation, but the discrete treatment is the same in math. If the potential distributions on the meridian flat are expressed as $(\mathrm{z}, \mathrm{r})$ in the flat field and axial symmetric field, the Laplace equation in flat field will be

$$
\frac{\partial^{2} V}{\partial z^{2}}+\frac{\partial^{2} V}{\partial r^{2}}=0
$$

and in rotational symmetric field

$$
\frac{\partial^{2} V}{\partial Z^{2}}+\frac{1}{r} \frac{\partial V}{\partial r}+\frac{\partial^{2} V}{\partial r^{2}}=0
$$

It can also be unified rewritten as

$$
\nabla^{2} V=\frac{\partial}{\partial z}\left(\beta \frac{\partial V}{\partial z}\right)+\frac{\partial}{\partial r}\left(\beta \frac{\partial V}{\partial r}\right)
$$

where $\beta=1$ means the flat field, and $\beta=\mathrm{r}$ means rotational symmetric field.

The continuous field in the solution area in $(\mathrm{z}, \mathrm{r})$ flat is scattered by the method dividing the region using the equidistant $(\mathrm{h} 1=\mathrm{h} 2)$ and unequidistant $(\mathrm{h} 1 \neq \mathrm{h} 2)$ grids constituted by the lines $\mathrm{z}= \pm \mathrm{ih} 1(\mathrm{i}=0,1,2, \ldots, \mathrm{m})$ and $\mathrm{r}= \pm \mathrm{jh} 2(\mathrm{j}=0,1,2, \ldots, \mathrm{n})$ which are parallel to coordinate axes. The intersection points of grid lines are known as nodes and the distance between two adjacent nodes are known as step. The continuous point set in solution area is turned to the discrete one, so that it can approximately replace the partial differential equations in continuous field by the corresponding difference equations. The potential values of nodes can be got by solving the difference equations by numerical calculation method and known as the approximate solution of (3). In this paper, the finite difference method is used to solve the equations as follows.

To establish five point difference format, as shown in Fig .1, the difference equations can be expanded by Taylor series without high-order items considering a point in solving region with coordinate $(\mathrm{z} 0, \mathrm{r} 0)$, voltage $\mathrm{V} 0$ and the distance to other adjacent points $h$, and the other four points' potential respectively V1, V2, V3, V4. The difference format is shown as

$$
\left\{\begin{array}{l}
\left(\frac{\partial V}{\partial z}\right)_{0}=\frac{V_{2}-V_{1}}{2 h} \\
\left(\frac{\partial V}{\partial r}\right)_{0}=\frac{V_{4}-V_{3}}{2 h} \\
\left(\frac{\partial^{2} V}{\partial z^{2}}\right)_{0}=\frac{V_{1}+V_{2}-2 V_{0}}{h^{2}} \\
\left(\frac{\partial^{2} V}{\partial r^{2}}\right)_{0}=\frac{V_{3}+V_{4}-2 V_{0}}{h^{2}}
\end{array}\right.
$$

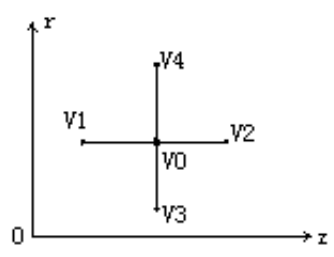

Figure 1. Isometric difference grid.
Laplace equation of rotational symmetric field in differential form can be got by feeding the (4) into the (2) as

$$
V_{1}+V_{2}+\left(1-h / 2 r_{0}\right) V_{3}+\left(1+h / 2 r_{0}\right) V_{4}-4 V_{0}=0\left(r_{0} \neq 0\right)
$$

where $r_{0}$ is the radial coordinates of $V_{0}$ (distance from axis of symmetry). The upper equation is only for the points in the solution region. For the points on the axis of symmetry, the upper equation can't be applied due to the conditions of $r_{0}=0$ and $\frac{1}{r}\left(\frac{\partial V}{\partial r}\right) \rightarrow \infty$, but the follows can be used:

$$
\lim _{r \rightarrow 0} \frac{1}{r}\left(\frac{\partial V}{\partial r}\right)=\frac{\partial^{2} V}{\partial r^{2}}
$$

to get the Laplace equation

$$
\frac{\partial^{2} V}{\partial z^{2}}+2 \frac{\partial^{2} V}{\partial r^{2}}=0
$$

By replacing the differential with difference, the equation can be expressed as

$$
V_{1}+V_{2}+4 V_{4}-6 V_{0}=0
$$

So to the any nodes the potential can be considering as

$$
\begin{gathered}
V_{i-1, j}+V_{i+1, j}+\left(1-\frac{h}{2 r_{0}}\right) V_{i, j-1}+\left(1+\frac{h}{2 r_{0}}\right) V_{i, j+1}-4 V_{i, j}=0,\left(r_{0} \neq 0\right),(9) \\
V_{i-1,0}+V_{i+1,0}+4 V_{i, 1}-6 V_{i, 0}=0 .\left(\mathrm{r}_{0}=0\right)
\end{gathered}
$$

For the n nodes' grid the equations are

$$
\sum_{j=1}^{n} a_{i, j} V_{j}=b_{i}(i=1,2, \cdots, n) .
$$

The matrix linear algebra equation group depends on discretization of grid nodes. for the numerical solution with enough accuracy, there are large numbers of nodes due to generally smaller value of step $h$, and the equations are solved by iteration method in common.

\section{B. Iterative method}

To solve the difference equations, the same time displacement iteration method, successive iteration method and successive over relaxation iteration method can be used. The same time displacement iteration method is unfavorable with very slow convergence rate and taking up more memory. The successive iteration method saves memory and accelerates the convergence rate.. Although the speed of the successive over relaxation iteration method can be greatly faster, the super relaxation factor is difficult to select. Considering the accuracy and speed of iterative method, successive iteration method is appropriate to solve the difference equations.

In successive iterative method, for the $(\mathrm{k}+1)$ th iteration, the potential of $(\mathrm{k}+1)$ th iteration is used not the kth 
iteration when considering the points over the $(k+1)$ th iteration. This iterative method saves the computer memory and accelerates the convergence rate. The equation for the successive iterative method is

$$
V_{\mathrm{i}}^{k+1}=\left\{b_{i}-\sum_{j<i} a_{i, j} V_{j}^{k+1}-\sum_{j>i} a_{i, j} V_{j}^{k}\right\} / \mathrm{a}_{\mathrm{i}, \mathrm{i}} .
$$

In five point difference, it is

$$
\begin{aligned}
& V_{\mathrm{i}, \mathrm{j}}{ }^{k+1}=\frac{1}{4}\left[V_{\mathrm{i}-1, \mathrm{j}}^{k+1}+V_{\mathrm{i}+1, \mathrm{j}^{k}}+\left(1-\frac{1}{2 j}\right) V_{\mathrm{i}, \mathrm{j}-1}^{k+1}\right. \\
& \left.+\left(1+\frac{1}{2 j}\right) V_{\mathrm{i}, \mathrm{j}+1}^{k}\right],\left(r_{\mathrm{i}, \mathrm{j}} \neq 0\right) \\
& V_{\mathrm{i}, 0}{ }^{k+1}=\frac{1}{6}\left[V_{\mathrm{i}-1,0}^{k+1}+V_{\mathrm{i}+1,0}{ }^{k}+4 V_{\mathrm{i}, 1}{ }^{k}\right]\left(r_{\mathrm{i}, 0}=0\right),
\end{aligned}
$$

where the residual error is defined as the potential difference between the adjacent two iterations as

$$
\varepsilon_{i}^{k+1}=V_{i}^{k+1}-V_{i}^{k}
$$

\section{Boundary and grid point processing}

First of all, divide the calculated region into equidistant and unequidistant grids in vertical and horizontal. The smaller grid step is taken up in the area where potential is faster or fine processing is required. If potential change is flat or fine processing is not required, you can use larger grid step.

Secondly, the grid points are divided into four types according to their properties. The normal point is in the interior of calculated area, and the surrounding four points which are the inner points of the whole step are involved in the iterations. Special point is in the interior of calculated area and nearby less than one whole step at the distance from boundary at least in one direction. Its iterative coefficients are calculated by the distance from boundary and grid step and nearby the boundary points are not involved in the iterations. Boundary point is on the boundary or whose distance is less than the given value from the boundary. The potential of boundary point is equal to the boundary's one and the boundary point is not involved in the iteration. External point is outside the boundary of the calculated region $G$. The potential of external point is equal to the boundary's one and the external point is not involved in the iteration.

Finally, the boundary should be enclosed. When the actual electrode system is opened, an approximate method can be used by broadening the electrodes to a certain extent as a fixed potential boundary. In potential gap position where the potential is changing, regard the axial potential is linearly changed. Next take the rotational symmetrical electrode system as a example. Add a supplement boundary with horizontal line along the $\mathrm{Z}$ direction between the equiradius of double cylinder electrodes. In case of the endpoint coordinates $\mathrm{za}$ and $\mathrm{zb}$, and endpoint potential $\mathrm{Va}, \mathrm{Vb}$, the potential of supplement boundary is calculated by linear interpolation as

$$
V(z)=V_{a}+\left(V_{b}-V_{a}\right)\left(z-z_{0}\right) /\left(z_{b}-z_{a}\right), \quad z_{a} \leq z \leq z_{b}
$$

For the equiradius add the complementary boundary along the $r$ direction perpendicular. In case of the endpoint coordinates ra and $\mathrm{rb}$, and endpoint potential $\mathrm{Va}, \mathrm{Vb}$, the potential of supplement boundary is calculated by logarithm interpolation as

$$
V(r)=V_{a}+\left(V_{b}-V_{a}\right) \ln \frac{r}{r_{a}} / \ln \frac{r_{b}}{r_{a}} \quad r_{a} \leq r \leq r_{b}
$$

By this way the errors are enough small.

\section{Calculation of the equipotential lines}

Derived from the theory, the equipotential lines on meridian plane satisfy the differential equations as follows:

$$
\left\{\begin{array}{l}
\frac{d r}{d s}= \pm \frac{\partial \phi}{\partial z} / \sqrt{\left(\frac{\partial \phi}{\partial r}\right)^{2}+\left(\frac{\partial \phi}{\partial z}\right)^{2}} \\
\frac{d z}{d s}=\mp \frac{\partial \phi}{\partial r} / \sqrt{\left(\frac{\partial \phi}{\partial r}\right)^{2}+\left(\frac{\partial \phi}{\partial z}\right)^{2}}
\end{array}\right.
$$

where $s$ is the arc length of the independent variables. The required equipotential lines should be expressed as

$$
r=r(\mathrm{~s}), z=z(\mathrm{~s}),
$$

which is called tracing method.

In fact, more simple and practical "scan-line searching method" can be used to calculate a series of points in equipotential lines independently. For the equipotential line of $\mathrm{Vd}$, line search along each transverse can be carried out to check the potential of every grid point. For example, scanning along the line $\mathrm{j}$, if $\mathrm{Vj}, \mathrm{i}$ is found less than $\mathrm{Vd}$ and $\mathrm{Vj}, \mathrm{i}+1$ is more than $\mathrm{Vd}$, the coordinates can be solved by linear interpolation between $\mathrm{Vj}, \mathrm{i}$ and $\mathrm{Vj}, \mathrm{i}+1$ as

$$
\left\{\begin{array}{l}
r_{d}=r_{j} \\
z_{d}=z_{i}+\left(z_{i+1}-z_{i}\right) \quad\left(V_{d}-V_{i, j}\right) /\left(V_{i+1, j}-V_{i, j}\right)
\end{array}\right. \text {. }
$$

Similarly, column scanning can be done along every column to draw the corresponding equipotential lines. Calculations show that the equipotential lines are smooth enough when the accuracy of iteration is high enough.

\section{PROGRAMMING AND RESULTS}

\section{A. Programming}

Based on the numerical calculation method, a program is designed to solve the field distributions and equipotential lines in a electrostatic immersion objective. Successive approximation method is used to solve the finite difference equation of field distributions and the scan-line searching method is used to solve the coordinate of equipotential lines. Immersion objective is also known as the cathode lens whose field distributions has been 
extended to the cathode plane of zero potential. In the electron beam tube immersion objective generally consists of three electrodes, cathode, modulation and accelerator. In imaging tube, it generally consists of two coaxial circular. All the structure of immersion objective is axial rotational symmetric. In a variety of fine beam tube, except for the geometrical size varied, immersion objective is almost the same in basic structure.

Structure of the electrostatic immersion objective are shown in Fig.2 with the variouses including the cathode potential of $\mathrm{Vc}$, modulation potential of $\mathrm{Vm}$, accelerator potential of $\mathrm{Vn}$, distance from modulation to cathode of $\mathrm{L} 1$, width of modulation of L2, distance from modulation to accelerator of L3, width of accelerator L4, length of supplement boundary of L5, distance from modulation to symmetric axis of $\mathrm{r} 1$, distance from modulation top to symmetric axis of $\mathrm{r} 2$, distance from accelerator bottom to symmetric axis of $\mathrm{r} 3$. The program design flow is divided into three modules, namely the initialization of program in Fig.3, an iterative solution in Fig.4 and solving equipotential lines in Fig.5.

Running the program, see the presentation of entering cathode potential of $\mathrm{Vc}$, modulation potential of $\mathrm{Vm}$, accelerator potential of $\mathrm{Vn}$ with the unit of $\mathrm{V}$. enter the parameters with "space" key as interval and "enter" key as end. Then enter the parameters related with boundary and electrodes following the notice. Also the grid size is required. With the entering parameters program takes the iterations and then prints the data of field distributions. After that, enter the potential of equipotential lines and end the program with chars " 12345 ".



Figure 2. Structure and variouses of the electrostatic immersion objective.

\begin{tabular}{|c|}
\hline defining a[t1][t2], $\mathrm{z}[\mathrm{t} 1], \mathrm{r}[\mathrm{t} 2], \mathrm{zd}[\mathrm{t} 1], \mathrm{rd}[\mathrm{t} 2], \mathrm{i}, \mathrm{j}, \mathrm{n}, \mathrm{et}$ al. \\
\hline Giving initial value to a[t1][t2] and grid size $\mathrm{d} 1, \mathrm{~d} 2$ \\
\hline Entering boundary conditions \\
\hline calculating L, S1, S5, S6, S7, S8 \\
$\mathrm{i}=0$ to $\mathrm{s} 1$ \\
$\mathrm{z}[\mathrm{i}]=\mathrm{i}^{*} \mathrm{~d} 1$ \\
$\mathrm{i}=0$ to $\mathrm{s} 2$ \\
$\mathrm{r}[\mathrm{i}]=\mathrm{i}^{*} \mathrm{~d} 2$ \\
$\mathrm{i}=0$ to $\mathrm{s} 2$ \\
$\mathrm{a}[0][\mathrm{i}]=\mathrm{Vc}$ \\
$\mathrm{i}=0$ to $\mathrm{s} 3$ \\
\hline $\mathrm{a}[\mathrm{i}][\mathrm{s} 2-1]=\mathrm{Vc}+(\mathrm{Vm}-\mathrm{Vc})^{*}(\mathrm{z}[\mathrm{i}]-\mathrm{z}[0]) /(\mathrm{z}[\mathrm{s} 3-1]-\mathrm{z}[0])$ \\
\hline initialization of boundary of modulation \\
$\mathrm{i}=\mathrm{s} 5-1$ to $\mathrm{s} 6$ \\
\hline $\mathrm{a} 6 \mathrm{i}][\mathrm{s} 2-1]=\mathrm{Vm}+(\mathrm{Vn}-\mathrm{Vm}) *(\mathrm{z}[\mathrm{i}]-\mathrm{z}[\mathrm{s} 5-1]) /(\mathrm{z}[\mathrm{s} 6-1]-$ \\
$\mathrm{z}[\mathrm{s} 5-1])$ \\
\hline initialization of boundary of accelerator $(\mathrm{Vn})$ \\
\hline
\end{tabular}

Figure 3. Flow chart of initialization of program.

\begin{tabular}{|c|c|c|}
\hline \multicolumn{3}{|c|}{ if $d>e$} \\
\hline \multicolumn{3}{|c|}{$\mathrm{d}=0$} \\
\hline \multicolumn{3}{|c|}{$\mathrm{i}=1$ to $\mathrm{s} 1-1$} \\
\hline \multicolumn{3}{|c|}{$\mathrm{j}=0$ to $\mathrm{s} 2-1$} \\
\hline \multicolumn{3}{|c|}{$\mathrm{j}=0$} \\
\hline true & \multicolumn{2}{|l|}{ false } \\
\hline \multirow{3}{*}{$\begin{array}{c}\mathrm{b}=(\mathrm{a}[\mathrm{i}-1][\mathrm{j}] \\
+\mathrm{a}[\mathrm{i}+1][\mathrm{j}] \\
+4 * \mathrm{a}[\mathrm{i}][\mathrm{j}+1]) \\
/ 6\end{array}$} & \multicolumn{2}{|c|}{ inner of boundary } \\
\hline & true & false \\
\hline & $\begin{array}{c}\mathrm{b}=(\mathrm{a}[\mathrm{i}-1][\mathrm{j}]+\mathrm{a}[\mathrm{i}+1][\mathrm{j}] \\
+(1-1 / 2 * \mathrm{j}) \mathrm{a}[\mathrm{i}][\mathrm{j}-1]+ \\
(1+1 / 2 * \mathrm{j})[\mathrm{i}][\mathrm{j}+1]) / 4\end{array}$ & $\mathrm{~b}=\mathrm{a}[\mathrm{i}][\mathrm{j}]$ \\
\hline \multicolumn{3}{|c|}{$|a[i][j]-b|>d$} \\
\hline true & \multicolumn{2}{|l|}{ false } \\
\hline \multicolumn{3}{|l|}{$\mathrm{d}=|\mathrm{a}[\mathrm{i}][\mathrm{j}]-\mathrm{b}|$} \\
\hline \multicolumn{3}{|c|}{$\mathrm{a}[\mathrm{i}][\mathrm{j}]=\mathrm{b}$} \\
\hline \multicolumn{3}{|c|}{ print the potential of nodes } \\
\hline \multicolumn{3}{|c|}{$\mathrm{i}=0$ to $\mathrm{s} 1$} \\
\hline \multicolumn{3}{|c|}{ print the axial potential a[i][0] } \\
\hline
\end{tabular}

Figure 4. Flow chart of iterative solution.

\begin{tabular}{|c|c|c|c|c|}
\hline \multicolumn{5}{|c|}{$V \mathrm{~d}=1$} \\
\hline \multicolumn{5}{|c|}{ if $V \mathrm{~d} \neq 12345$} \\
\hline \multicolumn{5}{|c|}{$\mathrm{N}=0$} \\
\hline \multirow{2}{*}{\multicolumn{5}{|c|}{$\begin{array}{l}\text { entering the potential of required equipotential lines } V \mathrm{~d} \\
\qquad V \mathrm{~d} \neq 12345\end{array}$}} \\
\hline & & & & \\
\hline \multicolumn{4}{|c|}{ true } & false \\
\hline \multicolumn{4}{|c|}{$\mathrm{j}=0$ to $\mathrm{s}_{2}$} & \\
\hline \multicolumn{4}{|c|}{$\mathrm{i}=0$ to $\mathrm{s}_{1}-1$} & \\
\hline \multicolumn{4}{|c|}{ outside of electrodes } & \\
\hline \multicolumn{3}{|c|}{ true } & false & \\
\hline \multicolumn{3}{|c|}{$V \mathrm{~d}$ between $\mathrm{a}[\mathrm{i}][\mathrm{j}]$ and $\mathrm{a}[\mathrm{i}+1][\mathrm{j}]$} & & \\
\hline true & \multicolumn{2}{|l|}{ false } & & \\
\hline \multirow{3}{*}{$\begin{array}{l}\text { calculating } \\
\mathrm{r}_{\mathrm{d}}[\mathrm{n}], \mathrm{z}_{\mathrm{d}}[\mathrm{n}]\end{array}$} & \multicolumn{2}{|c|}{$\mathrm{a}[\mathrm{i}][\mathrm{j}]=V \mathrm{~d}$} & & \\
\hline & true & false & & \\
\hline & $\mathrm{r}_{\mathrm{d}}[\mathrm{n}], \mathrm{z}_{\mathrm{d}}[\mathrm{n}], \mathrm{n}++$ & & & \\
\hline \multicolumn{5}{|c|}{$\mathrm{i}=0$ to $\mathrm{n}$} \\
\hline \multicolumn{5}{|c|}{ print the coordinate $\left(\mathrm{z}_{\mathrm{d}}[\mathrm{i}], \mathrm{r}_{\mathrm{d}}[\mathrm{i}]\right)$} \\
\hline
\end{tabular}

Figure 5. Flow chart of solving equipotential lines.

\section{B. Calculation example and result analysis}

The author considers the black and white imaging tube (type: $31 \mathrm{SX}-\mathrm{A}$ ) as example with the parameters of $\mathrm{Vc}=0 \mathrm{~V}, \mathrm{Vm}=-27 \mathrm{~V}, \mathrm{Vn}=120 \mathrm{~V}, \mathrm{~L} 1=0.13 \mathrm{~cm}, \mathrm{~L} 2=0.1 \mathrm{~cm}$, $\mathrm{L} 3=0.18 \mathrm{~cm}, \mathrm{~L} 4=0.2 \mathrm{~cm}, \mathrm{~L} 5=0.6 \mathrm{~cm}, \mathrm{r} 1=0.24 \mathrm{~cm}, \mathrm{r} 2=0.7 \mathrm{~cm}$ and $\mathrm{r} 3=0.24 \mathrm{~cm}$. Grid size is set at $\mathrm{d} 1=0.015 \mathrm{~cm}$ and $\mathrm{d} 2=0.025 \mathrm{~cm}$. The iterative precision of e is set at 0.001 . The distributions of axial potential and equipotential lines are achieved and shown in Fig .6 and 7 respectively. Results show that the rotationally symmetric electric field is characterized by nonuniformity so that the electron beam can be focused and imaged. Also axial potential distributions are nonlinear to produce a radial force and equipotential lines are distributed in a saddle on the nearby axial extreme point of potential. 


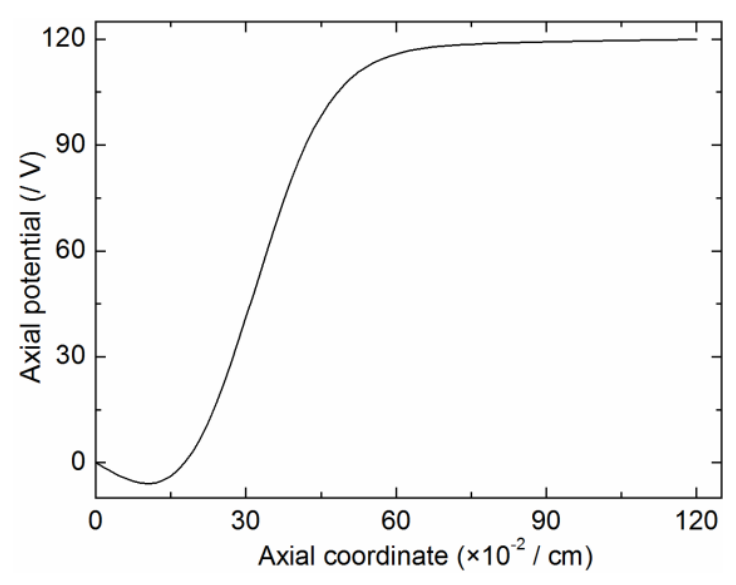

Figure 6. axial potential distributions.

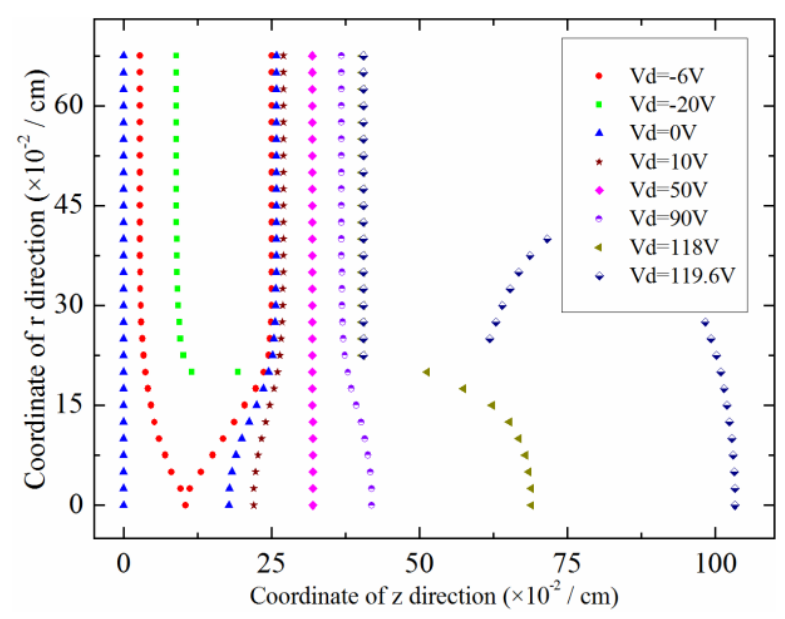

Figure 7. Distributions of equipotential lines.

\section{CONCLUSIONS}

Based on the numerical calculation method, a program is designed to solve the field distributions and equipotential lines in a electrostatic immersion objective. Successive approximation method is used to solve the finite difference equation of field distributions and the scan-line searching method is used to solve the coordinate of equipotential lines. In the calculation, the iterative precision of $\mathrm{e}$ is set at 0.001 which is enough to describe the field distributions, also it could be a higher precision.

Running the program results show that the rotationally symmetric electric field is characterized by nonuniformity so that the electron beam can be focused and imaged. Also axial potential distributions are nonlinear to produce a radial force and equipotential lines are distributed in a saddle on the nearby axial extreme point of potential.

\section{ACKNOWLEDGMENT}

This work is financially supported by the Natural Science Foundation of Jiangxi Province, China (Grant No. 20151BAB202019); the Youth Science Foundation of Jiangxi Province, China (Grant No. 20142BAB212007); and the Bidding Project of Gannan Normal University, China (Grant No. 14zb18).

\section{REFERENCES}

[1] M. Alshammari, K. Alshammari, A. Cudd, R. D. DuBois, and K. Tökési. "Exploring new aspects and practical applications of capillary guiding of charged particle beams. Secondary Exploring new aspects and practical applications of capillary guiding of charged particle beams," Nuclear Instruments and Methods in Physics Research Section B: Beam Interactions with Materials and Atoms, vol. 354, pp. 20-22, 2015.

[2] J. M. Mitrani, I. D. Kaganovich, and R. C. Davidson. "Mitigating chromatic effects on the transverse focusing of intense charged particle beams for heavy ion fusion. Secondary Mitigating chromatic effects on the transverse focusing of intense charged particle beams for heavy ion fusion," Nuclear Instruments and Methods in Physics Research Section A: Accelerators, Spectrometers, Detectors and Associated Equipment, vol. 733, pp. 65-69, 2014.

[3] Q. Zhang, K. Zhao, and Z. Chang. "High resolution electron spectrometers for characterizing the contrast of isolated 25 as pulses. Secondary High resolution electron spectrometers for characterizing the contrast of isolated 25 as pulses," Journal of Electron Spectroscopy and Related Phenomena, vol. 195, pp. 48-54, 2014.

[4] S. Ketelhut, L. J. Evitts, A. B. Garnsworthy, C. Bolton, G. C. Ball, R. Churchman, R. Dunlop, G. Hackman, R. Henderson, M. Moukaddam, E. T. Rand, C. E. Svensson, and J. Witmer. "Simulated performance of the in-beam conversion-electron spectrometer, SPICE. Secondary Simulated performance of the inbeam conversion-electron spectrometer, SPICE," Nuclear Instruments and Methods in Physics Research Section A: Accelerators, Spectrometers, Detectors and Associated Equipment, vol. 753, pp. 154-163, 2014.

[5] C. P. Aichele, D. Venkataramani, J. E. Smay, M. H. McCann, S. Richter, M. Khanzadeh-Moradllo, M. Aboustait, and M. T. Ley. "A comparison of automated scanning electron microscopy (ASEM) and acoustic attenuation spectroscopy (AAS) instruments for particle sizing. Secondary A comparison of automated scanning electron microscopy (ASEM) and acoustic attenuation spectroscopy (AAS) instruments for particle sizing," Colloids and Surfaces A: Physicochemical and Engineering Aspects, vol. 479, pp. 46-51, 2015.

[6] T. Yamada, K. Goushi, and A. Otomo. "Time-correlated single photon counting system and light-collection system for studying fluorescence emitters under high-vacuum conditions: Use of immersion objective and ionic liquid. Secondary Time-correlated single photon counting system and light-collection system for studying fluorescence emitters under high-vacuum conditions: Use of immersion objective and ionic liquid," Thin Solid Films, vol. 518, pp. 432-436, 2009.

[7] T. Velinov, Y. Asenovska, D. Marinkova, L. Yotova, S. Stoitsova, M. Bivolarska, and L. Stavitskaya. "Total internal reflection imaging of microorganism adhesion using an oil immersion objective. Secondary Total internal reflection imaging of microorganism adhesion using an oil immersion objective," Colloids and Surfaces B: Biointerfaces, vol. 88, pp. 407-412, 2011.

[8] A. Khursheed. "Ultimate resolution limits for scanning electron microscope immersion objective lenses. Secondary Ultimate resolution limits for scanning electron microscope immersion objective lenses," Optik - International Journal for Light and Electron Optics, vol. 113, pp. 67-77, 2002.

[9] M. Bernheim. "Image acquisition with immersion objective lenses using electrons emitted with several tenths of an electron volt energies: Towards high spatial resolution ESCA analysis. Secondary Image acquisition with immersion objective lenses using electrons emitted with several tenths of an electron volt energies: Towards high spatial resolution ESCA analysis," Ultramicroscopy, vol. 106, pp. 398-412, 2006.

[10] J. Jasny and J. Sepioł. "Single molecules observed by immersion mirror objective. A novel method of finding the orientation of a radiating dipole. Secondary Single molecules observed by immersion mirror objective. A novel method of finding the orientation of a radiating dipole," Chemical Physics Letters, vol. 273, pp. 439-443, 1997. 